\title{
Linx
}

Revue des linguistes de l'université Paris X Nanterre

\section{L'apprentissage des unités phonologiques variables : l'exemple du /R/ post-consonantique final en français}

Jean-Pierre Chevrot, Laurence Beaud et Renata Varga

\section{OpenEdition}

\section{Journals}

Édition électronique

URL : http://journals.openedition.org/linx/819

DOI : $10.4000 / \operatorname{linx} .819$

ISSN : 2118-9692

Éditeur

Presses universitaires de Paris Nanterre

\section{Édition imprimée}

Date de publication : 1 juin 2000

Pagination : 89-100

ISSN : 0246-8743

\section{Référence électronique}

Jean-Pierre Chevrot, Laurence Beaud et Renata Varga, «L'apprentissage des unités phonologiques variables : l'exemple du /R/ post-consonantique final en français », Linx [En ligne], 42 | 2000, mis en ligne le 22 juin 2012, consulté le 30 avril 2019. URL : http://journals.openedition.org/linx/819 ; DOI 10.4000/linx.819

Ce document a été généré automatiquement le 30 avril 2019.

Département de Sciences du langage, Université Paris Ouest 


\section{L'apprentissage des unités phonologiques variables : l'exemple $\mathrm{du} / \mathrm{R} /$ post-consonantique final en français}

Jean-Pierre Chevrot, Laurence Beaud et Renata Varga

1 Les descriptions de l'usage enfantin des variations phonologiques restent rares, particulièrement en France où le courant variationniste est peu représenté. Dans ce contexte, notre contribution prendra deux directions. Dans une première partie, un survol des études consacrées à l'usage enfantin des variantes phonologiques conduira à délimiter trois problématiques développementales. Dans une seconde partie, le compterendu d'une expérimentation décrivant l'apprentissage du / $/$ / post-consonantique final ( quat'sous, liv'd'images, etc.) chez des enfants de 8-9 ans fournira des données susceptibles d'alimenter deux des questions théoriques: l'influence de l'orthographe sur l'apprentissage des unités phonologiques variables et la nature du dispositif - règle ou autre - qui réalise cet apprentissage.

\section{Trois problématiques développementales}

2 Un premier axe de débat est une interrogation sur l'âge d'apparition, chez l'enfant, des " patterns » sociolinguistiques observés chez l'adulte (effet de la situation, de l'origine sociale, etc.). Bien que discuté depuis trois décennies (Labov 1972; Patterson 1992; Roberts 1997, etc.), ce point n'a fait l'objet d'aucun consensus, en raison d'imprécisions qui brouillent le débat. D'abord, l'âge auquel les régularités se mettent en place dépend de la saillance perceptive des variantes en jeu, de leur nature articulatoire et de leur valeur sociolinguistique dans chaque communauté. Ensuite, les conditions méthodologiques des enquêtes ne sont pas assez standardisées pour assurer la fiabilité des comparaisons. Enfin, le questionnement lui-même est empreint d'adultocentrisme, les auteurs ne s'intéressant 
aux variations enfantines qu'à partir du moment où elles prennent la même forme que chez l'adulte.

Une seconde interrogation concerne les relations entre la variabilité qui intéresse les linguistes et la variabilité développementale mise en évidence dans les mots enfantins par les psycholinguistes. Il est possible qu'une variation résiduelle héritée de la période d'acquisition soit recyclée dans le changement de style (Ingram et al. 1985). Ainsi, la variabilité précoce échapperait en partie aux contraintes articulatoires et cognitives et serait mise au service d'objectifs sociaux.

4 La troisième et dernière problématique se décline sous la forme de quatre questions sur la dynamique de l'acquisition des unités phonologiques variables.

51 - Quel est le mécanisme par lequel les enfants encodent des unités variables dans leur compétence phonologique en cours de construction?

6 Labov (1989) et Roberts (1997) suggèrent que des enfants de 3-4 ans construisent une connaissance abstraite de la variation, sous la forme d'une règle variable. Toutefois, Wolfram (1988) constate un conditionnement lexical de la variation enfantine peu compatible avec la généralisation qu'implique une règle. Cette question rencontre un débat similaire dans le champ psycholinguistique. En effet, des propositions théoriques inspirées du connexionnisme remettent l'accent sur la capacité des apprentis à extraire des contraintes probabilistes de l'environnement, sans qu'il soit nécessaire d'invoquer la construction de règles abstraites pour en rendre compte (Seidenberg 1997; Laks 1996). Ces conceptions renvoient dos à dos les défenseurs des règles variables et leurs détracteurs qui doutaient de la capacité de l'esprit humain à intérioriser des probabilités.

72 - Les facteurs linguistiques des variations résultent-ils de l'apprentissage de traits dialectaux ou de l'émergence de contraintes articulatoires et cognitives universelles?

8 Roberts (1997) et Labov (1989) soutiennent l'hypothèse de l'apprentissage dialectal. La tendance des enfants anglophones à supprimer plus souvent (-t,-d) devant consonne que devant voyelle s'accentuant avec l'âge, Roberts en conclut que ce facteur est appris. Toutefois, des données issues du français (Chevrot 1991) relativisent ces conclusions en montrant la précocité et la force de l'impact de l'environnement phonologique sur la suppression $\mathrm{du} / \mathrm{R} /$ post-consonantique final.

9 3-Quel dynamisme développemental règle l'apparition des facteurs sociaux de la variation?

10 Selon Labov (1972), les dispositions stylistiques n'apparaîtraient qu'à l'adolescence, lorsque le locuteur découvre l'éventail et la valeur sociale des formes linguistiques. Cette supposition est mise en doute par des enquêtes montrant que certaines adaptations stylistiques sont acquises dès 3-4 ans (Patterson 1992). De plus, les études sur le développement métalinguistique (Gombert, 1990) établissent que l'enfant de 3-4 ans module la forme de ses énoncés par des modifications comportementales globales, peu conscientes, associées automatiquement aux situations. En bref, des habiletés stylistiques pratiques précéderaient la conscience de la signification sociale des usages.

114 - Quel est le rôle du contact scolaire avec la forme écrite des mots dans l'acquisition des unités phonologiques variables?

12 Une série de résultats (Chevrot 1991) suggère que l'apprentissage de la forme écrite de la locution conjonctive "parce que" conduit les jeunes locuteurs francophones à ajouter un /R/ à la représentation phonologique de ce mot. Bien que les études systématiques sur ce 
point soient rares, les faits diachroniques confirment l'éventualité de cette influence de l'orthographe sur la prononciation (Buben 1935).

\section{L'apprentissage du /R/ post-consonantique final}

\section{Une expérimentation?}

En fondant ses avancées sur des enquêtes réalisées dans le milieu habituel des locuteurs, la linguistique de la variation a favorisé la validité externe de sa démarche. Ce choix rencontre des limites lorsque la vérification d'hypothèses rend nécessaire un contrôle plus serré des variables. Ainsi, Labov (1972) et d'autres ont-ils utilisé des expérimentations pour établir le rôle de l'attention et du feed-back auditif. L'apprentissage des unités phonologiques variables compte parmi les questions qui nécessitent des procédures d'observation garantissant une meilleure validité interne.

\section{Une expérimentation conçue pour tester trois hypothèses}

Selon notre première hypothèse, le contact avec la forme écrite de certains mots permettrait aux jeunes locuteurs de mettre à jour des représentations phonologiques lexicales incomplètes, où manque un phonème très souvent supprimé dans l'environnement linguistique oral, tel le /R/ de «parce que ».

Selon notre seconde hypothèse, la représentation phonologique de mots rares appris à travers la lecture et l'écriture serait déduite d'une représentation orthographique stable. Leur prononciation doit donc être plus stable et les suppressions phonémiques moins fréquentes, quels que soient les facteurs sociolinguistiques, articulatoires ou perceptifs qui favorisent la variation.

Enfin, l'expérimentation étant fondée sur l'apprentissage de pseudo-mots contenant un / $\mathrm{R} /$ post-consonantique final susceptible de suppression (dorénavant $\mathrm{CR} \#$ ), elle nous renseignera sur la façon dont le dispositif cognitif responsable de la variation traite les mots nouveaux ${ }^{1}$. La question cruciale est de savoir si ce dispositif généralisera la variabilité à tout nouvel item lexical comportant CR\#.

\section{Le /R/ final post-consonantique : un marqueur sociolinguistique}

Depuis trois siècles, les commentaires sur l'usage attestent l'existence de cette variante et témoignent de sa valeur sociale péjorative (Laks 1980). Des observations plus récentes auprès d'enfants et d'adolescents (Laks 1980; Chevrot, 1991) établissent que la suppression covarie avec des différences sociologiques, qu'elle est plus fréquente devant consonne que devant voyelle ou pause, et plus fréquente en situation d'entretien que lors de la lecture d'un texte.

\section{Procédure expérimentale}

18 Quarante huit enfants de troisième année primaire (8-9 ans), répartis aléatoirement dans trois groupes, ont appris trois pseudo-mots masculins contenant CR\# et désignant des animaux imaginaires représentés sur des dessins. Dans le matériel verbal présenté aux enfants, ces items étaient prononcés [kasõt $(\mathrm{R})]$, [bidz̃ $\mathrm{k}(\mathrm{R})]$, [malop(R)] et ils précédaient 
un adjectif commençant par la consonne /b/. Les formes écrites correspondantes étaient quaçontre, bydeincre, maullopre ${ }^{2}$. Ces trois items comportent 9 lettres et manifestent une structure orale de type $\mathrm{C}_{1} \mathrm{VC}_{2} \mathrm{VC}_{3} \mathrm{R}$, où $\mathrm{C}_{3}$ est une occlusive sourde. L'apprentissage, réalisé individuellement sur 4 séances réparties sur 4 demi-journées successives, a pris des formes différentes dans chacun des trois groupes de 16 enfants. Chaque enfant du groupe «oral » a appris les pseudo-mots par le canal oral uniquement, en écoutant deux types d'enregistrements : description d'un paysage mettant en scène les animaux imaginaires et dénomination d'images. Dans chacun des enregistrements, chaque pseudo-mot était présenté avec /R/ maintenu dans $25 \%$ des occurrences entendues, et supprimé dans les $75 \%$ restants. Le groupe « oral-écrit » écoutait les mêmes enregistrements que le groupe «oral» pendant les deux premières séances. A partir de la troisième séance, cette audition était doublée d'un contact avec la graphie des pseudo-mots, qui contient la lettre " $\mathrm{r}$ ", lors de tâches de copie et de lecture silencieuse avec le même matériel verbal que les enregistrements. Les sujets du groupe "écrit» ont appris les mots par l'écrit uniquement. Dès la première séance, ils manipulaient leur graphie par la copie et la lecture silencieuse. Chacun des 48 enfants rencontrait 8 fois chaque item dans chacune des quatre séances d'apprentissage.

19 A la fin de chaque séance d'apprentissage, chaque enfant participait à deux tâches de production orale destinées à enregistrer les progrès dans l'apprentissage de la représentation phonologique des pseudo-mots. Dans un premier temps, il devait produire 16 occurrences de chacun des items, par dénomination orale des images représentant les animaux imaginaires. Les occurrences produites étaient insérées à l'environnement C-\#C, qui favorise la suppression, la consonne de droite étant un /b/ (bydeincre blanc / bleu). Dans un deuxième temps, chaque enfant syllabait deux fois chaque pseudo-mot. Cette tâche est censée favoriser la réalisation de toutes les unités présentes dans la représentation phonologique.

\section{Echantillon et population}

Les 48 locuteurs participant à l'expérience sont issus de deux populations scolaires contrastées $^{3}$. La moitié d'entre eux fréquentait un groupe scolaire situé dans une Z.E.P, l'autre moitié l'école d'un secteur résidentiel. La population de la première école compte $30 \%$ de parents ouvriers et $10 \%$ de parents cadres supérieurs. Les mêmes proportions sont respectivement de $10 \%$ et de $40 \%$ dans la seconde école. Dans chacun des trois groupes « oral ", " oral-écrit » et " écrit », 8 sujets provenaient de la première école et 8 de la seconde. Ces indications sur les populations scolaires sont aussi des informations sur la constitution sociologique des familles et des groupes de pairs. Nous vérifierons ainsi si nos patrons généraux de résultats se maintiennent chez des sujets participant à des environnements sociolectaux différents.

\section{Audibilité des maintiens et des suppressions}

21 Les enregistrements proposés à l'écoute des sujets ont été réalisés par un adulte produisant sur commande suppressions et maintiens de /R/ dans les trois pseudo-mots. Nous avons vérifié si les variantes visées étaient bien les variantes perçues. Quatre enfants de chacune des deux écoles, de la même fourchette d'âge que les 48 sujets de l'expérience, ont participé à un test d'audibilité. Lors d'une écoute individuelle, 
l'expérimentateur demandait aux auditeurs de répéter immédiatement chaque occurrence de la variante du pseudo-mot entendue. Sur 192 réponses, nous n'avons constaté que 2 erreurs. Suppressions et maintiens de /R/ sont donc parfaitement audibles par des enfants centrant leur attention sur les variantes.

\section{Résultats : l'influence de l'orthographe sur l'apprentissage de CR\#}

Les passations ont donné lieu à 384 enregistrements des tâches de dénomination et de syllabation. Pour la tâche de dénomination, nous avons calculé un pourcentage individuel de conservation rapportant le nombre de formes avec un [R] final au nombre total de formes produites. Ce pourcentage, établi pour chacune des tâches de dénomination suivant les trois dernières séances d'apprentissage ${ }^{4}$, est calculé sur 48 occurrences par sujet, obtenues en confondant les 3 pseudo-mots. S'agissant de la syllabation, nous avons évalué, pour chaque mot et pour chacune des trois dernières sessions, un score individuel selon le critère suivant: 1 si au moins une forme syllabée d'un item sur les deux jets demandés comporte un $[R], 0$ sinon. Les enfants syllabant trois pseudo-mots, ce score varie entre 0 et 3 . Les moyennes par groupe et par séance sont présentées dans le tableau 1 (dénomination) et le tableau 2 (syllabation).

Tableau 1 - Tâche de dénomination, moyenne des pourcentages individuels de conservation de /R/ à l'issue des trois dernières séances d'apprentissage

\begin{tabular}{|l|l|l|l|}
\hline max. = 100 & & & \\
\hline & après séance 2 & après séance 3 & après séance 4 \\
\hline \hline groupe « oral » & 48 & 51 & 49 \\
\hline groupe « oral-écrit » & 26 & 78 & 79 \\
\hline \hline groupe « écrit » & 86 & 92 & 91 \\
\hline
\end{tabular}

Tableau 2 - Tâche de syllabation, moyenne des scores individuels de conservation de /R/ à l'issue des trois dernières séances d'apprentissage

\begin{tabular}{|l|l|l|l|}
\hline max. = & & & \\
\hline & après séance 2 & après séance 3 & après séance 4 \\
\hline groupe « oral " & 1,6 & 1,7 & 1,7 \\
\hline groupe « oral-écrit " & 0,8 & 2,6 & 2,3 \\
\hline \hline groupe « écrit » & 2,5 & 2,7 & 2,7 \\
\hline
\end{tabular}

23 L'application de tests statistiques permet de déceler les différences entre moyennes dont la significativité est inférieure au seuil usuel de 0,05. S'agissant de la tâche de dénomination (tableau 1), les pourcentages de conservation des groupes «oral» et 
" écrit » ne manifestent aucune évolution significative de la séance 2 à la séance 4 . En revanche, les valeurs du groupe "oral-écrit " augmentent significativement entre la séance 2 et la séance 3, moment où l'écrit est introduit, puis marquent un plateau entre la séance 3 et la séance 4 . Dès la séance 2 , on constate en outre que les taux du groupe «écrit » sont proches de la valeur maximale de $100 \%$ de conservation de /R/, alors que ceux du groupe «oral » fluctuent autour de $50 \%$. En bref, seul le groupe "oral-écrit » progresse, en conservant davantage le $/ \mathrm{R} /$ à partir du moment où il rentre en contact avec la graphie. Si le groupe «écrit » ne progresse pas, c'est parce qu'il est en contact avec les graphies dès le début de l'apprentissage et qu'il atteint, dès la séance 2, des performances presque maximales.

24 Cette configuration reste identique pour les moyennes de la tâche de syllabation (tableau 2) et pour les enfants issus des deux environnements sociolectaux, entre lesquels n'apparaît d'ailleurs aucune différence significative. Les deux hypothèses concernant l'influence de l'orthographe sur l'apprentissage des unités phonologiques variables sont donc confirmées.

\section{Résultats : capacité de généralisation du dispositif qui encode la variation}

Le calcul des moyennes du tableau 1 confond les pseudo-mots et masque la réponse à une question capitale. Soit ces moyennes proviennent de pourcentages individuels de conservation généralement différents de 0 et de 100 pour chaque mot. Si tel est le cas, un même locuteur a produit le même pseudo-mot avec et sans [R] lors de la même session. Il faut en conclure qu'il a encodé un /R/ variable lors de l'apprentissage. Soit ces moyennes proviennent de pourcentages individuels généralement égaux à 0 ou à 100 pour chaque mot. Dans ce cas, il faut conclure que les locuteurs ont appris une des deux variantes phonétiques de surface: celle qui inclut un $[\mathrm{R}]$ ou celle qui en est démunie. Nous observerons cette question à travers les données du tableau 3, où le nombre de locuteurs ayant obtenu soit 0 , soit 100 , soit un score intermédiaire est noté par mot, par groupe et par séance.

Bien que les enfants des groupes « oral » et « oral-écrit» aient tous entendu la variante avec $[\mathrm{R}]$ et la variante sans $[\mathrm{R}]$ de chaque pseudo-mot, leurs pourcentages de conservation par item valent 0 ou 100 dans environ $81 \%$ des cas de ce tableau. Dans $19 \%$ des cas seulement, la variation des productions suggère que les locuteurs ont encodé la variabilité lors de l'apprentissage. De leur côté, les enfants du groupe «écrit » n'ont eu qu'un contact avec la forme écrite des pseudo-mots, où la lettre « $r$ » est stable. Bien qu'ils n'aient jamais perçu un input auditif variable, ils produisent marginalement un /R/ variable dans $11 \%$ des cas. Malgré ce dernier résultat, la tendance massive semble être de mémoriser une des variantes, plutôt que d'encoder un /R/ variable. Cette tendance se maintient d'ailleurs dans les deux environnements sociolectaux.

Tableau 3 - Nombre de locuteurs selon le pourcentage de conservation par pseudo-mot : 0,100 , autre valeur.

\begin{tabular}{|l|l|l|l|l|}
\hline & Groupe « oral » & groupe « oral-écrit » & groupe « écrit » \\
\hline
\end{tabular}




\begin{tabular}{|c|c|c|c|c|c|c|c|c|c|}
\hline & $\begin{array}{l}\text { séance } \\
2\end{array}$ & $\begin{array}{l}\text { séance } \\
3\end{array}$ & $\begin{array}{l}\text { séance } \\
4\end{array}$ & $\begin{array}{l}\text { séance } \\
2\end{array}$ & $\begin{array}{l}\text { séance } \\
3\end{array}$ & $\begin{array}{l}\text { séance } \\
4\end{array}$ & $\begin{array}{l}\text { séance } \\
2\end{array}$ & $\begin{array}{l}\text { séance } \\
3\end{array}$ & $\begin{array}{l}\text { séance } \\
4\end{array}$ \\
\hline \multicolumn{10}{|c|}{ quaçontre } \\
\hline 0 & 8 & 7 & 4 & 7 & 2 & 2 & 1 & 1 & 2 \\
\hline 100 & 6 & 5 & 6 & 3 & 11 & 12 & 13 & 15 & 14 \\
\hline autre & 2 & 4 & 5 & 3 & 3 & 1 & 1 & 0 & 0 \\
\hline \multicolumn{10}{|c|}{ maullopre } \\
\hline 0 & 9 & 8 & 7 & 14 & 3 & 4 & 2 & 0 & 1 \\
\hline 100 & 4 & 4 & 5 & 1 & 7 & 9 & 9 & 12 & 13 \\
\hline autre & 3 & 4 & 4 & 1 & 6 & 3 & 4 & 4 & 2 \\
\hline \multicolumn{10}{|c|}{ bydeincre } \\
\hline 0 & 6 & 4 & 6 & 8 & 2 & 2 & 1 & 1 & 1 \\
\hline 100 & 9 & 8 & 9 & 4 & 10 & 12 & 12 & 13 & 14 \\
\hline autre & 1 & 4 & 1 & 3 & 3 & 2 & 2 & 2 & 1 \\
\hline
\end{tabular}

\section{Discussion générale et conclusion}

Les principales tendances sont compatibles avec les deux hypothèses relatives à l'influence de l'orthographe sur la variation de /R/. Les résultats du groupe "écrit » suggèrent qu'une prononciation déduite d'une représentation orthographique tend à une stabilité qui se manifeste par la non-variabilité de phonèmes pourtant supprimables dans d'autres mots. Ce résultat répond à la question de Dell (1982) sur l'apprenabilité des exceptions lexicales à la règle optionnelle d'effacement, posée pour rendre compte de la chute des liquides post-consonantiques finales. En effet, un enfant qui entend un mot où le / $\mathrm{R} /$ est conservé ne peut pas décider s'il s'agit de la non application ordinaire d'une règle optionnelle, ou bien de la manifestation ponctuelle du statut d'exception de ce mot. En fait, il est probable que les mots rares, qui justement sont ceux qui dérogent à la chute de $/ R /$, soient manipulés ou appris sous leur forme écrite. Ce simple fait suffirait à expliquer leur particularité, sans avoir à supposer que les jeunes locuteurs repèrent les exceptions à une règle abstraite.

Les résultats concernant le groupe "oral-écrit» indiquent que l'apprentissage de l'orthographe d'un mot permet aux jeunes locuteurs d'ajouter, à sa représentation lexicale, un phonème souvent supprimé dans l'environnement oral. Cette incidence de l'orthographe sur la phonologie agit immédiatement, dès la séance 3 , après qu'a eu lieu le contact avec la graphie. Dans les modèles psycholinguistiques, l'apprentissage de l'orthographe est décrit, entre autres, comme l'établissement de connexions entre unités 
phonologiques et unités orthographiques, qui deviennent susceptibles de se mobiliser par un mécanisme d'activation interactive. C'est ce mécanisme qui mettrait l'information orthographique mémorisée au service de la production orale (Chevrot et Malderez, 1999).

Un troisième résultat concerne la tendance des enfants à privilégier une des variantes entendues, plutôt que d'encoder directement un /R/ variable. Chevrot (1991) a constaté que, dès 6-7 ans au moins, les enfants possédaient un dispositif - règle variable ou autre produisant l'effacement de CR\#. Notre procédure expérimentale ne prétend donc pas simuler la période de construction d'un tel dispositif. Elle reproduit plutôt la situation où des mots nouveaux sont présentés à l'entrée d'un dispositif déjà construit. Et le fait est que ce dispositif ignore ces nouveaux items chez une majorité d'enfants, dont on peut résumer ainsi le comportement: soit ils n'ont pas encodé /R/ dans la représentation lexicale, et [R] n'apparaît jamais dans la production; soit ils l'ont encodé, et il est catégoriquement présent. Il est donc improbable que le dispositif en question puisse être représenté par une règle s'appliquant systématiquement à tout élément du lexique comportant la configuration requise. Nos résultats suggèrent au contraire que la potentialité de variation est une propriété lexicale, acquise mot par mot par un contact prolongé avec les différentes variantes. Il reste que ce dispositif manifeste toutefois des capacités limitées de généralisation, comme l'attestent les cas minoritaires où un /R/ variable est encodé.

Il faut noter enfin qu'aucune différence sociolectale ne se manifeste dans l'usage oral des mots nouvellement appris. Ce résultat suggère que le mécanisme qui apprend et qui traite la variation est le même pour tous. Toutefois, les enfants des différents milieux rencontrant les deux variantes avec des fréquences différentes, ils les encoderont avec des poids distincts et auront plus ou moins tendance à supprimer le /R/. Si on neutralise ces différences dans « l'input », comme le fait notre expérimentation, alors les différences disparaissent aussi dans « l'output ».

31 Finalement, ces données interrogent la position de Roberts (1997) et de Labov (1989), qui soutiennent que les enfants de 3-4 ans construisent une règle variable en réponse à un input variable. La raison d'être d'une règle est de devenir de plus en plus abstraite (Pacton, soumis à publication). On voit donc mal comment une règle déjà construite pourrait avoir perdu à 8-9 ans des capacités de généralisation lexicale qu'elle possédait à 3-4 ans, alors qu'elle était en construction. Si nos hypothèses sont exactes, l'acquisition précoce de CR\# doit débuter par l'apprentissage de la variante sans [R], les enfants de 2 ans ayant une forte tendance à tronquer les groupes obstruente-liquide (Vinter, à paraître). La rencontre répétée avec les deux variantes et la maturation articulatoire conduiraient l'enfant à encoder et produire un $/ \mathrm{R} /$ variable. Finalement, pour quelques mots, la mise à jour des formes lexicales s'achèverait tardivement, lors de la manipulation des graphies.

\section{BIBLIOGRAPHIE}

BUBEN, V. (1935). Influence de l'orthographe sur la prononciation du français moderne. Droz. 
CHEVROT, J.-P. (1991). La variation phonétique chez des enfants de 6 à 12 ans : aspects développementaux et incidence des apprentissages alphabétiques, doctorat. Grenoble 3.

CheVrot, J.-P. \& MALDEREZ, I. (1999). «L'effet Buben : de la linguistique diachronique à l'approche cognitive (et retour) ». Langue Française, 104-125.

DeLL, F. (1982). « On gaps undetectable for language learners ». In J. Mehler, E. C.T. Walker \& M. Garett (eds.), Perspectives in mental representation, L.E.A., 431-438.

GOMBERT, J.-E. (1990). Le développement métalinguistique. P.U.F.

Ingram, J., Pittam, J. \& Newman, D. (1985). « Developmental and Sociolinguistic Variation in the Speech of Brisbane Schoolchildren ». Australian Journal of Linguistics 5(2), 233-246.

LABov, W. (1972). Sociolinguistics Patterns. Philadelphia, University of Pennsylvania Press (trad.fr. (1976). Sociolinguistique. Editions de Minuit).

LABOV W. (1989). « The Child as Linguistic Historian ». Language Variation and Change, 1(1), 85-97.

LAKS, B. (1980). Différenciation linguistique et différenciation sociale : quelques problèmes de sociolinguistique française, Doctorat de $3^{\text {ième }}$ cycle, Paris 8.

LAKS, B. (1996). Langage et cognition. Hermès.

PACTON, S. (soumis à publication). «Implicit Learning of Graphotactic and Morphonological Constraints: Evidence from Children and Adults Nonword Judgements of Plausibility ».

PATTERSON, J. (1992). The development of sociolinguistic phonological variation patterns for (ing) in young children. Ph. D. University of New Mexico.

ROBERTS, J. (1997). « Acquisition of variable rules: a study of (-t, d) deletion in preschool children ». Journal of Child Language, 24, 351-72.

SEIDENBERG, M.S. (1997). « Language Acquisition and Use: Learning and Applying Probalistic Constraints ». Science, 275, 1599-603.

VINTER, S. (à paraître). « Le développement phonologique de l'enfant de 2 ans ». Paroles.

Wolfram, W. (1989). «Structural Variability in phonological Development: Final Nasals in Vernacular Black English ». In R. W. Fasold \& D. Schriffrin (ed.), Language Variation and Change, John Benjamins Publishing Company, 301-32.

\section{NOTES}

1. Nous utilisons des pseudo-mots contenant un $/ \mathrm{R} /$ final plutôt que des vrais mots afin de nous assurer qu'il s'agit bien d'éléments lexicaux nouveaux pour tous les sujets. Par ailleurs, ce choix permet un meilleur contrôle du matériau phonique : nombre de segments, consonne avant /R/, etc.

2. Chaque item contient deux graphèmes non-déductibles de la mise en oeuvre des correspondances graphoniques les plus probables. Cette disposition ne sera pas exploitée ici.

3. Ce travail n'aurait pas abouti sans la patience des enseignants et des directrices des groupes scolaires où nous avons recueilli nos données. Qu'ils en soient remerciés.

4. Un trop grand nombre de non-réponses interdit le calcul d'un tel score à l'issue de la première séance d'apprentissage. 


\section{RÉSUMÉS}

La variation phonologique a été largement décrite dans le cadre de la sociolinguistique variationniste. En dépit d'avancées indéniables fondées sur l'observation d'adultes ou d'adolescents, les approches développementales de la variation restent rares. Dans ce contexte, nous commencerons par une brève revue des travaux disponibles, qui permettra d'identifier trois problématiques développementales débattues dans ce champ. Nous présenterons ensuite une expérimentation qui éclaire certaines des questions théoriques en examinant le mécanisme impliqué dans l'apprentissage $\mathrm{du} / \mathrm{R} /$ post-consonantique final du français (liv'd'image, quat'sous, etc.) chez des enfants de 8-9 ans. Les résultats suggèrent deux conclusions : (1) les enfants ont tendance à copier les formes de surface rencontrées dans l'environnement linguistique plutôt qu'à encoder une règle variable (2) l'acquisition des graphies déclenche l'encodage tardif de certains /R/ dans les représentations lexicales.

Phonological variation has been the object of many studies conducted in the field of sociolinguistic research. Despite undeniable advances which are based on the observation of adults and adolescents, developmental approaches to variation remain rare. In this context, this article starts with a review which identifies three theoretical problem areas in the field of research into phonological variation in children. We then present an experimental study which supply data which can help us shed light on some of these issues. This experiment examines the mechanism involved in the learning of variable word-final post-consonantal /R/ in French ( pauv'petit, liv'd'image, etc.) in 8 to 9 years old children. The results suggest two conclusions: (1) children have a tendency to copy surface forms rather than encode a variable rule (2) spelling acquisition causes the late encoding of certain /R/s in the lexical representations.

\section{AUTEURS}

\section{JEAN-PIERRE CHEVROT}

Lidilem, GDR Phonologies

Université Stendhal

BP 25, 38040

Grenoble CEDEX 09, France.

chevrot@icp.inpg.fr

LAURENCE BEAUD

Rennes 2

RENATA VARGA

Lidilem 